\title{
The large intracellular loop of ptch1 mediates the non-canonical Hedgehog pathway through cyclin B1 in nevoid basal cell carcinoma syndrome
}

\author{
FEI-YAN YU ${ }^{1}$, YING-YING HONG ${ }^{1}$, JIA-FEI QU ${ }^{1}$, FENG CHEN $^{2}$ and TIE-JUN LI ${ }^{1}$ \\ ${ }^{1}$ Department of Oral Pathology and ${ }^{2}$ Central Laboratory, \\ Peking University School and Hospital of Stomatology, Haidian District, Beijing 100081, P.R. China
}

Received January 16, 2014; Accepted May 6, 2014

DOI: $10.3892 / \mathrm{ijmm} .2014 .1783$

\begin{abstract}
Mutations in the transmembrane receptor patched homolog 1 (Homo sapiens) (ptch1) are responsible for nevoid basal cell carcinoma syndrome (NBCCS), an autosomal dominant disorder that causes developmental abnormalities and predisposes the affected individuals to cancer. Many of these mutations, including mutations in the $\mathrm{C}$-terminus of the large intracellular loop (ICL) of ptch1 (p.C727VfsX745 and p.S733IfsX736), result in the premature truncation of the protein. The ptch1-C727VfsX745 and ptch1-S733IfsX736 mutations have been identified in patients with NBCCS-associated keratocystic odontogenic tumors (KCOTs). In the present study, we found that the molecular mechanisms regulated by the noncanonical Hedgehog (Hh) signaling pathway through cyclin B1 are involved in the pathogenesis of NBCCS-associated KCOTs. In contrast to wild-type ptch1, ptch1-C727VfsX745 and ptch1-S733IfsX736 clearly exhibited reduced binding to cyclin B1. Moreover, the cells expressing these two mutations demonstrated an increase in cell cycle progression and these two mutation constructs failed to inhibit cell proliferation. In addition, the mutants enhanced the activity of gliomaassociated oncogene family zinc finger 1 (GLI1), a downstream reporter of Hh signaling. Thus, our data suggest that the noncanonical Hh pathway mediated through ptch1 and cyclin B1 is involved in the pathogenesis of NBCCS-associated KCOTs. The C-terminus of ICL in ptch1 may also be a potential therapeutic target in the treatment of this disease.
\end{abstract}

Correspondence to: Dr Feng Chen, Central Laboratory, Peking University School and Hospital of Stomatology, 22 South Zhongguancun Avenue, Haidian, Beijing 100081, P.R. China

E-mail: moleculecf@gmail.com

Professor Tie-Jun Li, Department of Oral Pathology, Peking University School and Hospital of Stomatology, 22 South Zhongguancun Avenue, Haidian, Beijing 100081, P.R China

E-mail: litiejun22@vip.sina.com

Key words: nevoid basal cell carcinoma syndrome, non-canonical Hedgehog pathway, cyclin B1, ptch1

\section{Introduction}

Nevoid basal cell carcinoma syndrome (NBCCS; also known as Gorlin-Goltz syndrome; OMIM no. 109400), which was first reported by Gorlin and Goltz in 1960 (1), is an autosomal dominant disorder $(2,3)$. NBCCS is characterized by a wide range of developmental abnormalities and a predisposition to neoplasms, such as multiple keratocystic odontogenic tumors (KCOTs), bifid ribs, dyskeratotic palmar and plantar pits, progressive falcine calcification, vertebral and shoulder abnormalities, as well as basal cell carcinoma (2-6).

Currently, the pathogenesis of this complex disorder is thought to be the consequence of germline mutations in the patched homolog 1 (Homo sapiens) (PTCH1) gene (OMIM no. 601309) (7-11). $P$ TCH1, which was originally discovered in 1996 , has been mapped to chromosome 9q22.3 and consists of 23 exons. The PTCH1 gene encodes a glycoprotein (ptch1) of containing 1,447 amino acids, which form 12 transmembrane regions, 2 large extracellular loops (ECLs), and a large intracellular loop (ICL) (12-14). The ptch1 protein is essential for embryonic development, and controlling cellular proliferation and fate $(12,13,15)$. Moreover, through its role as a receptor in the Hedgehog $(\mathrm{Hh})$ signaling pathway, ptch1 provides a molecular basis for NBCCS.

ptch1 is a multifunctional protein containing a variety of functional domains (Fig. 1A) (16). Based on these various functional domains of ptch1 and the 280 germline mutations extensively distributed throughout the domain structure of the ptch 1 protein $(8,17)$, it is possible that these mutations contribute to the various functions of ptch1 and may be used to identify the molecular mechanisms regulated by Hh signaling. However, studies have not yet demonstrated whether mutations in the ICL of ptch1 are involved in the pathogenesis of NBCCS-associated KCOTs. The C-terminus of ICL in ptch1 indicates a putative mutation hot-spot region $(8,17)$. Two $P T C H 1$ frameshift mutations, i.e., c.2179delT (p.C727VfsX745) and c.2196_2197delCT (p.S733IfsX736), within this region introduce the premature truncation of the ptch1 protein (Fig. 1B), which is identified in patients with NBCCS-associated KCOTs $(11,14,18-20)$. The nucleotide numbering was based on GenBank entry NM_000264.3. The amino acid numbering was based on GenBank entry NP_000255.2. 
According to current reports, Hh signaling proceeds through canonical and non-canonical pathways, and a large number of genetic and biochemical studies has established the canonical Hh pathway in vertebrates $(16,21,22)$. Although non-canonical Hh signaling is not yet fully understood, it has been shown that ptch1 participates in a novel $\mathrm{G}_{2} / \mathrm{M}$-phase checkpoint, independent of other Hh pathway components (16). During the late $\mathrm{G}_{2}$ phase, cyclin $\mathrm{B} 1$ and cdc2 form the regulatory and catalytic subunits of the mitosis-promoting factor (MPF) complex (23-25). The activation of the MPF is regulated by the phosphorylation of 4 serine residues (Ser126, -128, -133 and -144) in the cytoplasmic retention signal (CRS) of cyclin B1, which results in the nuclear localization of MPF, promoting cell cycle progression (26-30). Barnes et al demonstrated that the large ICL of human ptch1 specifically interacts with the constitutively phosphorylated cyclin B1 in basal cell carcinoma (BCC) through the non-canonical Hh pathway $(31,32)$. However, the evidence supporting the involvement of non-canonical $\mathrm{Hh}$ signaling in NBCCS-associated KCOTs is not conclusive.

Therefore, the aim of this study was to elucidate the mechanisms regulated by non-canonical Hh pathway associated with ptch1 and cyclin B1 in NBCCS-associated KCOTs in order to gain a better understanding of this disease. Our findings suggest that the non-canonical Hh pathway is involved in the pathogenesis of NBCCS-associated KCOTs.

\section{Materials and methods}

Expression constructs. The ptch1-C727VfsX745 and ptch1-S733IfsX736 mutation constructs (Fig. 1B) were created using a QuikChange II XL Site-Directed Mutagenesis kit (Agilent Technologies, Inc., Beijing, China) and an N-terminal HA-tagged PTCH1 plasmid (EX-T0559-M06; GeneCopoeia Inc., Rockville, MD, USA) as the template, according to the manufacturer's instructions. The primers for the mutants were designed online (www.genomics.agilent. com). The pCMX-cyclin B1 plasmid, containing an N-terminal Myc tag (Addgene, Cambridge, MA, USA), served as a template to construct the cyclin $\mathrm{B} 1^{\mathrm{Glu}}$ derivative with the $4 \mathrm{Ser}$ phosphorylation sites in the CRS domain mutated to Glu to mimic a phosphorylated state. We synthesized CRS ${ }^{\mathrm{Glu}}$ and its derivative modified by appending a nuclear localization signal (NLS, NLS-CRS ${ }^{\mathrm{Glu}}$ ), and then inserted it into the pcDNA3.1 vector (Invitrogen Life Technologies Corp., Carlsbad, CA, USA) $(28,31)$. All constructs were verified by DNA sequencing.

Cell proliferation assays. Human embryonic kidney 293T cells, originally obtained from ATCC (Manassas, VA, USA) and maintained in our laboratory, were seeded at a density of $1.0 \times 10^{5}$ cells/plate $(60 \mathrm{~mm})$ and transfected using Lipofectamine 2000 (Invitrogen Life Technologies Corp.) with $4.0 \mathrm{mg}$ of empty HA vector (mock), ptch1 (wild-type), ptch1C727VfsX745 or ptch1-S733IfsX736 DNA. The cells were cultured in Dulbecco's modified Eagle's medium (DMEM; Gibco/Invitrogen Life Technologies Corp.) with $10 \%$ fetal bovine serum (FBS; HyClone Laboratories, Inc., South Logan, UT, USA). Following transfection, we counted the cells every 24 h using a Coulter Counter (Beckman Coulter Inc., Brea, $\mathrm{CA}, \mathrm{USA})$. The results represent the means $\pm \mathrm{SD}$ of 3 separate transfections.
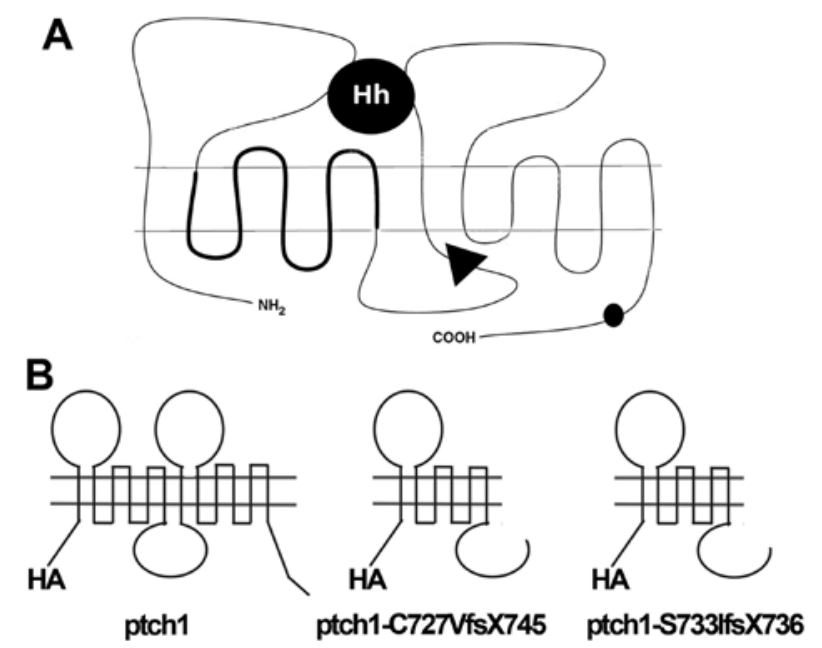

Figure 1. Functional domains in ptch1 and schematic representation of ptch1, ptch1-C727VfsX745 and ptch1-S733IfsX736 constructs. (A) Functional domains in ptch1. Hh morphogens interact with the 2 large extracellular loops (ECLs) of ptch1. Transmembrane domains 2-6 comprise the sterol-sensing domain (SSD; thick line). The C-terminal of intracellular loop (ICL) contains the cyclin B1 interaction domain (black triangle). The C-terminal cytoplasmic tail contains an addiction/dependence domain (ADD; black circle). (B) Schematic representation of ptch1 and ptch1 mutations. Ptch1-C727VfsX745 is a frameshift mutation located in the large ICL, and introduces the premature truncation of the ptch1 protein at amino acid residue 745 . The frameshift mutation ptch1-S733IfsX736 results in a stop codon at residue 736 within the large ICL of ptch1. Each construct contains an N-terminal HA tag.

Glioma-associated oncogene family zinc finger 1 (GLII)luciferase reporter assays. NIH3T3 cells (preserved in our laboratory) grown to $80 \%$ confluence in 24 -well plates were co-transfected with $4 \mathrm{mg}$ of GLI1-luciferase reporter construct (Genentech Inc., San Francisco, CA, USA) and $2 \mathrm{mg}$ of the empty HA vector (mock), ptch1, ptch1-C727VfsX745, or ptch1-S733IfsX736 constructs using Lipofectamine 2000. The cells were serum-starved with $0.2 \%$ FBS for another $24 \mathrm{~h}$, after which the cells were lysed. Luciferase activity was measured using the Dual-Luciferase Reporter Assay System (Promega Corp., Madison, WI, USA) at 2 days after transfection. Each assay was performed multiple times, and the results shown represent the means \pm SD of 3 independent experiments, presented as GLI1-luciferase reporter activity in the transfected cells.

Western blot analysis. At $48 \mathrm{~h}$ after transfection, the cells were rinsed with phosphate-buffered saline (PBS) and then lysed in TNE lysis buffer (10 mMTris-HCL, pH 7.5, $150 \mathrm{mMNaCl}$, $1 \mathrm{mM}$ EDTA, 0.5\% NP 40) containing PhosSTOP Phosphatase Inhibitor Cocktail (Roche Diagnostics, Indianapolis, IN, USA) and Complete Protease Inhibitor Cocktail (Roche Diagnostics) for $30 \mathrm{~min}$ on ice. Generally, cells from one $10-\mathrm{cm}$ dish were lysed with $0.5 \mathrm{ml}$ TNE buffer. The samples were spun at $14,000 \mathrm{x} \mathrm{g}$ for $15 \mathrm{~min}$ at $4^{\circ} \mathrm{C}$, and the protein content was standardized using the Bradford protein assay kit (Applygen Technologies Inc., Beijing, China). The samples were analyzed by sodium dodecyl sulfate polyacrylamide gel electrophoresis (SDS-PAGE) on $10 \%$ gels, transferred to nitrocellulose membranes, and immunoblotted for Myc-cyclin B1, HA-tagged ptch1, and HA-tagged mutated ptch1 using anti-Myc-tag 
(9B11) antibodies (Cell Signaling Technology, Beverly, MA, USA) and anti-HA-tag (C29F4) antibodies (Cell Signaling Technology), each at a 1:1,000 dilution. All samples were probed with the appropriate fluorescein-conjugated secondary antibodies, either mouse or rabbit, at a dilution of 1:15,000. GAPDH was used as a loading control where appropriate.

Flow cytometric analysis. The $293 \mathrm{~T}$ cells were seeded at $80 \%$ confluence in $60-\mathrm{mm}$ dishes. At $36 \mathrm{~h}$ after transfection with the empty vector, ptch1, ptch1-C727VfsX745 or ptch1-S733IfsX736 constructs, the 293T cells were harvested, washed twice with PBS, and fixed in $70 \%$ ethanol overnight at $4^{\circ} \mathrm{C}$. The fixed cells were washed with PBS prior to incubation with $50 \mu \mathrm{g} / \mathrm{ml}$ RNase and $0.1 \%$ Triton X-100 in PBS for $30 \mathrm{~min}$ at $37^{\circ} \mathrm{C}$. The cells were then stained with propidium iodide (PI) to facilitate the analysis of cell cycle distribution using a FACScan laser flow cytometer (FACSCalibur; BectonDickinson, Franklin Lakes, NJ, USA).

Co-immunoprecipitation (CoIP). At $36 \mathrm{~h}$ after transfection, the 293T cells were rinsed with PBS and then lysed in TNE buffer. Following centrifugation $\left(14,000 \mathrm{x} \mathrm{g}, 15 \mathrm{~min}, 4^{\circ} \mathrm{C}\right)$, the cell extracts were pre-cleared with protein A/G PlusAgarose beads (Santa Cruz Biotechnology, Santa Cruz, CA, USA) and then incubated with Myc-tag (9B11) antibodies at a dilution of 1:1,000 and the appropriate Protein A/G PlusAgarose (Santa Cruz Biotechnology) overnight at $4^{\circ} \mathrm{C}$. The samples were washed with TNE buffer and resuspended in SDS-PAGE loading buffer. Finally, the samples were analyzed by SDS-PAGE on $10 \%$ gels, transferred to nitrocellulose membranes, and immunoblotted for Myc-cyclin B1 and HA-tagged ptch1 proteins using Myc-tag (9B11) or HA-tag (C29F4) antibodies, each at a 1:1,000 dilution. Western blot analysis was performed using standard procedures.

Confocal immunofluorescence. Two days after transfection using Lipofectamine 2000, COS-1 cells, obtained from ATCC, were fixed with $4 \%$ paraformaldehyde, permeabilized with $0.2 \%$ Triton X-100 and blocked with 5\% FBS in PBS. Triple immunofluorescence labeling was performed as follows: i) Myc-tagged CRS ${ }^{\mathrm{Glu}}$ and NLS-CRS ${ }^{\mathrm{Glu}}$ derivatives were detected with Myc-tag (9B11) antibodies and Alexa Fluor 555 Conjugate (Cell Signaling Technology); ii) HA-tagged ptch1, HA-tagged ptch1-C727VfsX745, or HA-tagged ptch1-S733IfsX736 construct expression was detected using anti-HA-tag (C29F4) antibodies and Alexa Fluo 488 Conjugate (Cell Signaling Technology); iii) nuclei were stained with 4',6-diamidino-2-phenylindole (DAPI). The cells were detected using the laser confocal scanning microscope imaging system.

Statistical analysis. Data were analyzed using SPSS software (version 13.0), and significant differences between numerical groups were determined using the independent t-test. P-values $<0.05$ were considered to indicate statistically significant differences. All statistical tests were two-sided.

\section{Results}

ptch1-C727VfsX745 and ptch1-S733IfsX736 mutation constructs fail to inhibit cell proliferation. We examined the effects of the $2 \mathrm{PTCH} 1$ mutations, which introduce premature truncations within the large ICL of ptch1 protein (Fig. 1B), using cell proliferation assays in $293 \mathrm{~T}$ cells transiently transfected with the appropriate constructs (Fig. 2A). Transfection with ptch1 (wild-type) significantly decreased cell numbers by 15-33\% compared with the mock-transfected cells. Moreover, transfection with ptch1-C727VfsX745 or ptch1-S733IfsX736 markedly increased cell numbers by $32-64 \%$ and $30-49 \%$, respectively, at 2-4 days following transfection, as compared with the ptch1-transfected cells, but only marginally increased cell numbers by $8-20 \%$ or $4-12 \%$, respectively, compared with the mock-transfected cells. These data suggest that the overexpression of wild-type ptch1 inhibited cell growth, which is consistent with the hypothesis that ptch1 acts as a tumor suppressor (32). However, the expression of the mutant constructs, ptch1-C727VfsX745 and ptch1-S733IfsX736, did not lead to the inhibition of cell proliferation. Importantly, our results revealed that ptch1 and the mutant proteins were successfully expressed in the $293 \mathrm{~T}$ cell lysates, as shown in the inset of Fig. 2A.

Expression of ptch1-C727VfsX745 or ptch1-S733IfsX736 enhances GLII activity, consistent with the activation of the Hh signaling pathway. The overexpression of wild-type ptch1 did not increase GLI1 activity compared with the mock-transfected cells. By contrast, ptch1-C727VfsX745 and ptch1-S733IfsX736 expression markedly increased GLI1 reporter activity compared with the ptch1-transfected cells by 106 and $99 \%$, respectively (Fig. 2B). These results demonstrated that the expression of these 2 ptch 1 mutant proteins induced GLI1 reporter activity, thereby activating intracellular signaling associated with the Hh pathway.

$293 T$ cells expressing ptch1-C727VfsX745 or ptch1S733IfsX736 proteins progress rapidly through the $G_{2} / M$ phase of the cell cycle. To confirm the effects of these 2 mutations on the activity of cyclin B1, we performed flow cytometric analysis to determine whether these mutants affect the cell cycle distribution. In the 293T cells overexpressing ptch1-C727VfsX745 or ptch1-S733IfsX736, the percentage of cells in the $G_{2}$ phase decreased by approximately 5 - or 3 -fold compared to the ptch1-transfected cells (Fig. 2C). These results suggested that the ptch1 mutants promoted the rapid progression of the 293T cells through the $\mathrm{G}_{2} / \mathrm{M}$ phase of the cell cycle.

Mutations in the large ICL of ptchl reduce binding to cyclin B1. In order to determine whether ptch1-C727VfsX745 or ptch1-S733IfsX736 are associated with cyclin B1, we performed co-immunoprecipitation experiments. We found that neither ptch1 mutant constructs co-immunoprecipitated with cyclin $\mathrm{B} 1^{\mathrm{Glu}}$ well. By contrast, wild-type ptch1 bound to

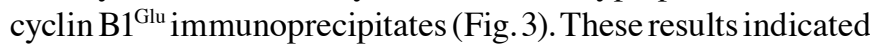
that the prematurely truncated mutants, ptch1-C727VfsX745 and ptch1-S733IfsX736, exhibited reduced binding with phosphorylated cyclin B1 as compared with wild-type ptch1.

ptch1 mutation in the C-terminus of ICL does not alter the localization of cyclin B1. The ptch1, ptch1-C727VfsX745 and ptch1-S733IfsX736 proteins were localized to the membrane (Fig. 4A). The CRS ${ }^{\text {Glu }}$ entered the nucleus (Fig. 4Ba 
A

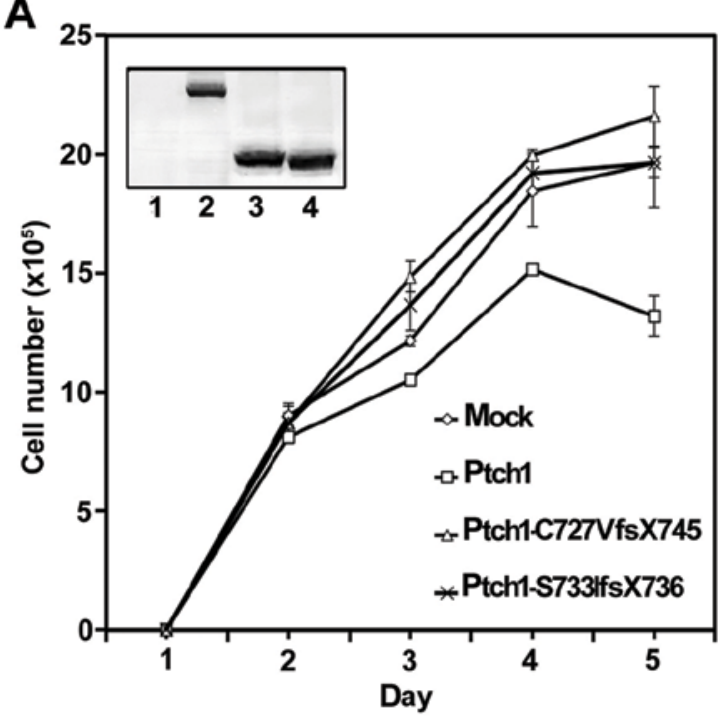

C

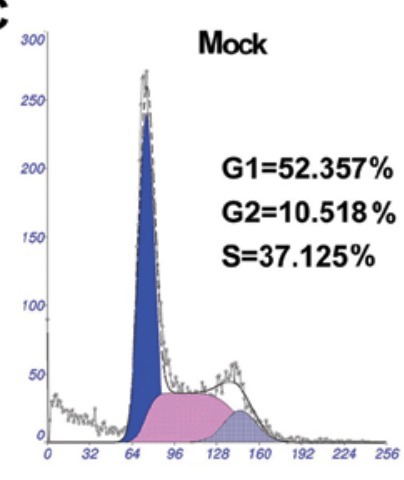

DNA content

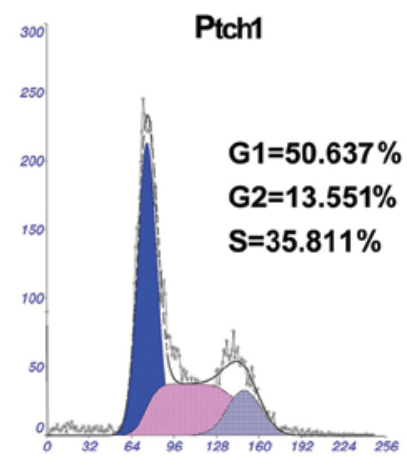

DNA content
B
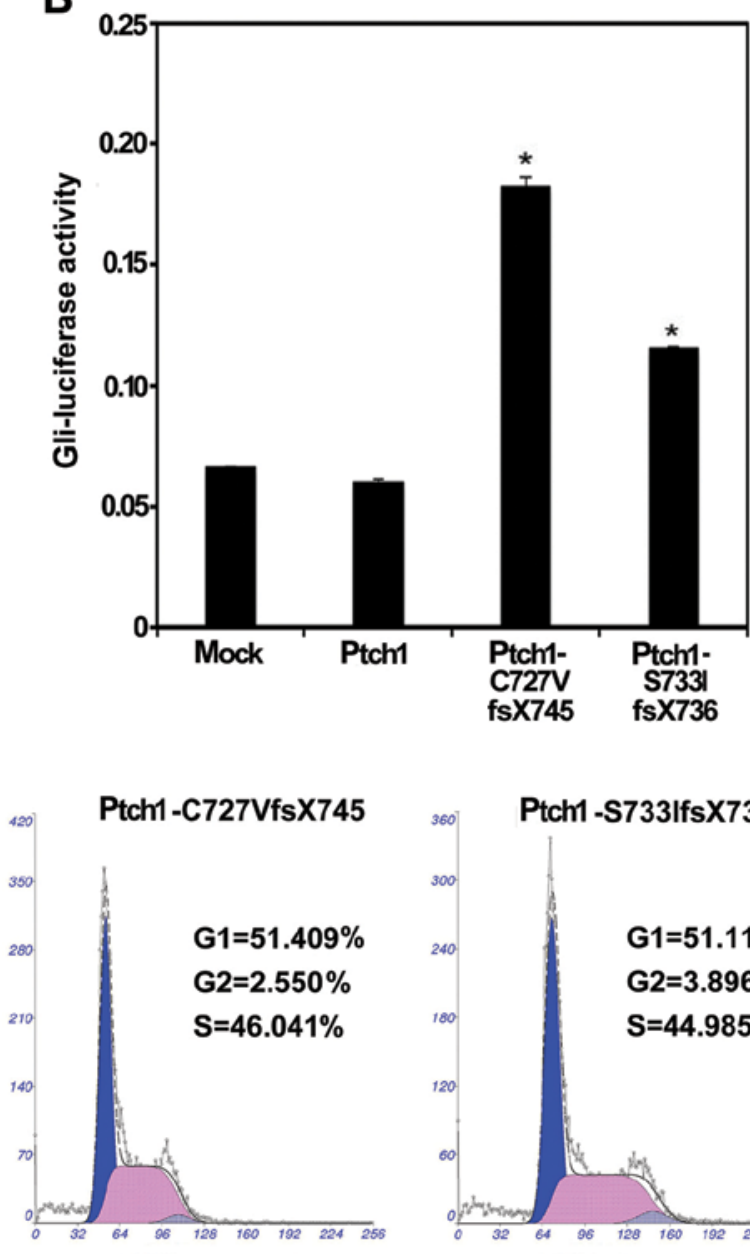

DNA content

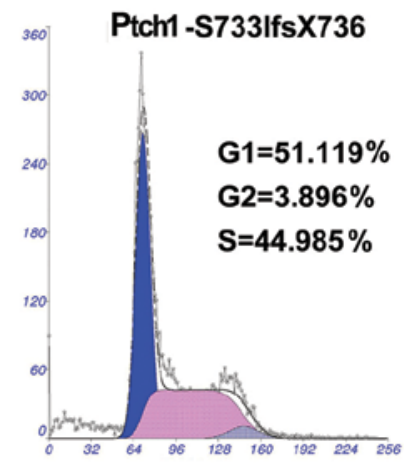

DNA Content

Figure 2. The ptch1 mutation in the C-terminus of the intracellular loop (ICL) affects the biological functions of the ptch1 protein. (A) ptch1-C727VfsX745 and ptch1-S733IfsX736 were unable to inhibit cell proliferation. 293T cell growth curves for cells expressing mock, ptch1, ptch1-C727VfsX746 or ptch1-S733IfsX736 vectors. Inset: expression of HA-tagged ptch1 and -mutated ptch1 proteins in cell lysates from day 3 (sample 1, mock; sample 2, ptch1; sample 3, ptch1-C727VfsX745; sample 4, ptch1-S733IfsX736). (B) Expression of ptch1-C727VfsX745 and ptch1-S733IfsX736 constructs enhanced GLI1 activity. NIH3T3 cells were co-transfected with empty HA vector, HA-ptch1, HA-ptch1-C727VfsX746, or HA-ptch1-S733IfsX736 and a GLI1-luciferase reporter construct. At 2 days after transfection, cell lysates were subjected to luciferase assays. The expression of these 2 mutations increased the GLI1 reporter activity compared with that in ptch1-transfected cells. All results are representative of 3 independent experiments; ${ }^{2}<0.05$. (C) Cellular expression of ptch1C727VfsX745 and ptch1-S733IfsX736 promoted a rapid progression through the $\mathrm{G}_{2} / \mathrm{M}$ phase of the cell cycle. We transfected 293T cells with the empty vector (mock), HA-tagged ptch1, HA-tagged ptch1-C727VfsX745, or HA-tagged ptch1-S733IfsX736 constructs and measured the cell cycle distribution after $36 \mathrm{~h}$. The percentage of cells in the $\mathrm{G}_{2}$ phase significantly decreased in the cells transfected with the 2 mutation constructs compared to the ptch1-transfected cells. Each experiment was performed independently 3 times.

and b), and NLS-CRS Glu was constitutively localized to the nucleus (Fig. 4Bc and d).

As endogenous ptch1 expression levels in the COS-1 cells are low, we co-transfected these cells with an empty vector (mock), HA-tagged ptch1, HA-tagged ptch1-C727VfsX745 or HA-tagged ptch1-S733IfsX736 constructs with the NLS-CRS ${ }^{\text {Glu }}$ construct to determine whether the ptch1 mutations in the C-terminus of ICL alter its subcellular localization. NLS-CRS ${ }^{\text {Glu }}$ exhibited altered localization to the membrane when co-expressed with ptch1 (Fig. 4Ce-h). By contrast, NLS-CRS ${ }^{\text {Glu }}$ retained its nuclear localization in the presence of ptch1-C727VfsX745 and ptch1-S733IfsX736 (Fig. 4Ci-p). Thus, these data suggest that ptch1-C727VfsX745 and ptch1S733IfsX736 do not alter the localization of phosphorylated cyclin B1.

\section{Discussion}

Although ptch1 plays an important role in the regulation of $\mathrm{Hh}$ signaling in a variety of cellular processes, the biological relevance of the different functional domains of ptch1 in relation to the molecular mechanisms of non-canonical Hh signaling remains to be fully understood. In this study, we demonstrated that ptch1 mutations in the cyclin B1 interaction domain reduced binding with the phosphorylated form of cyclin B1 and prevented the recruitment of cyclin B1, thereby regulating the nuclear localization of the MPF complex and promoting cell cycle progression. These mutations also activated the Hh signaling pathway and accelerated cell proliferation. Therefore, it is possible that the obvious proliferative activity of KCOTs, compared with other odontogenic cysts, may be 

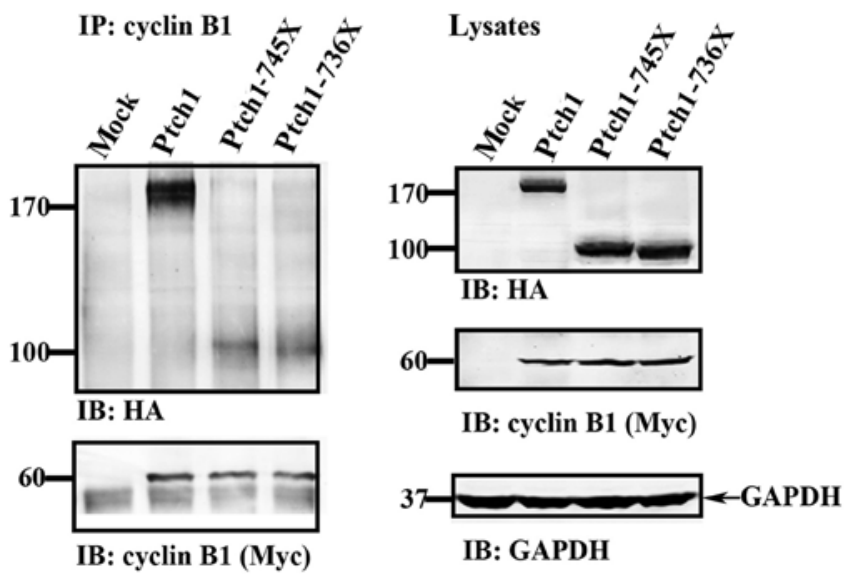

Figure 3. Ptch1-C727VfsX745 and ptch1-S733IfsX736 show reduced binding to cyclin $\mathrm{B}^{\mathrm{Glu}}$. The $293 \mathrm{~T}$ cells were co-transfected with the empty HA vector, HA-ptch1, HA-ptch1-C727VfsX746, or HA-ptch1-S733IfsX736 and Myc-tagged cyclin B1 ${ }^{\text {Glu }}$ constructs. Cell lysates were immunoprecipitated with anti-Myc-tag antibodies to examine ptch1 by immunoblot analysis. Upper left panel: interaction between cyclin $\mathrm{B} 1^{\mathrm{Glu}}$ immunoprecipitates and mock (lane 1), ptch1 protein (lane 2), ptch1-C727VfsX745 (lane 3) and ptch1-S733IfsX736 (lane 4). Lower left panel: equal immunoprecipitation of cyclin $\mathrm{B}^{\mathrm{Glu}}$ (lanes 2-4). Upper right panel: immunoblot analysis of ptch1 protein in mock- (lane 1), ptch1- (lane 2), ptch1-C727VfsX745- (lane 3) and ptch1S733IfsX736- (lane 4) transfected cells. Center right panel: equal expression of cyclin $\mathrm{B} 1^{\mathrm{Glu}}$ proteins (lanes 2-4). Lower right panel: equal protein loading using anti-GAPDH antibodies (lanes 1-4). Co-immunoprecipitation was performed independently at least 3 times and representative blots are shown.

associated with the activity of the non-canonical Hh pathway through the regulation of cyclin B1.

Ptch1 is a putative tumor suppressor frequently mutated in association with NBCCS $(15,20,33)$. Approximately $9 \%$ of germline mutations are distributed in the ICL of the ptch1 protein $(8,17)$; therefore, this domain, comprising residues $599-750$, may be identified as a mutation hot spot region $(8,17)$. However, there is little evidence to support that mutations in this domain are involved in the pathogenesis of NBCCS-associated KCOTs. In the present study, we found that the p.C727VfsX745 and p.S733IfsX736 mutations within this region participated in the regulation of the nuclear localization of MPF and promoted cell growth. In this manner, ptch1 required the C-terminus of ICL (727-745) to bind to cyclin B1, and mutations in this region reduced the ability of ptch1 to bind to cyclin B1. Additionally, the subcellular localization of the MPF complex is controlled by the C-terminus of ICL, and mutations in this region alter the subcellular localization of the MPF complex, resulting in the loss of ptch1 anti-oncogenic function. Thus, the C-terminus of ICL may be important for ptch1 function.

NBCCS-associated KCOT treatment requires a multidisciplinary approach, including surgical removal, laser ablation, photodynamic therapy and chemotherapy $(4,20)$. However, these methods do not provide favorable long-term positive outcomes $(34,35)$. Based on the functional domains of the ptch1 protein, appropriate treatments may vary among patients with different mutations. In the current study, we found that mutations in the C-terminus of the ICL altered the nuclear localization of the MPF complex and promoted cell proliferation. Therefore, the C-terminus of the ICL may be a potential therapeutic target in the treatment of this disease.

The activation of GLI1 is an important cellular response to the aberrant activation of the $\mathrm{Hh}$ signaling pathway $(16,21,36,37)$. In the present study, we found that the p.C727VfsX745 and p.S733IfsX736 mutations activated the Hh signaling cascade. However, the canonical Hh pathway can still explain the consequences of disrupted Hh signaling. Our
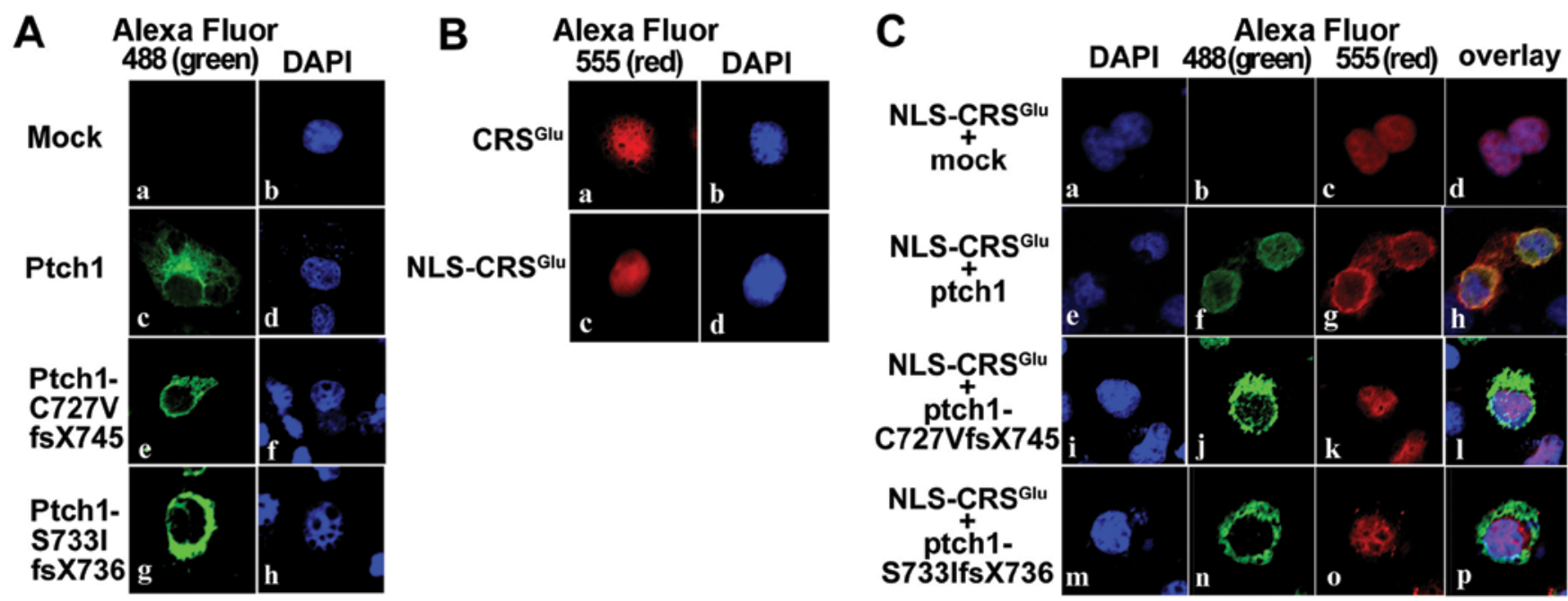

Figure 4. The ptch1 mutation in the C-terminus of the intracellular loop (ICL) does not alter the localization of the nuclear localization signal (NLS) \pm cytoplasmic retention signal (CRS) ${ }^{\text {Glu }}$. (A) Localization of HA-tagged ptch1, ptch1-C727VfsX745 and ptch1-S733IfsX736 in COS-1 cells detected by confocal immunofluorescence. (a) No expression detected in mock. (c, e and g) Cells expressing ptch1, ptch1-C727VfsX746 or ptch1-S733IfsX736 vectors at the membrane. (b, d, f and h) Nuclei detected with DAPI. (B) Localization of human CRS ${ }^{\text {Glu }}$ and NLS-CRS Glu in COS-1 cells detected by confocal immunofluorescence. (a and c) CRS $^{\text {Glu }}$ and NLS-CRS ${ }^{\text {Glu }}$ expressed in the nucleus. (b and d) Nuclei detected with DAPI. (C) Localization of NLS \pm CRS Glu upon co-transfection with ptch1 or ptch1 mutation constructs. (b-d) NLS $\pm \mathrm{CRS}^{\mathrm{Glu}}$ showed nuclear localization with empty HA vector expression. (f-h) Localization of NLS \pm CRS ${ }^{\mathrm{Glu}}$ at the membrane due to co-transfection of ptch1. (j-1 and n-p) By contrast, NLS \pm CRS ${ }^{\text {Glu }}$ retained the nuclear localization in the presence of ptch1-C727VfsX745 and ptch1-S733IfsX736. (a, e, i and m) Nuclei detected with DAPI. 
study suggests that non-canonical pathways may be involved in the occurrence and progression of NBCCS-associated KCOTs. Indeed, our data suggest that certain cellular processes are regulated by non-canonical pathways, canonical pathways, or both. Further studies are required to fully elucidate the precise molecular mechanisms mediating these events.

In conclusion, we analyzed and identified the molecular mechanisms regulated by non-canonical Hh signaling through the cyclin B1 interaction domain in ptch1. Our data demonstrate that the $\mathrm{C}$-terminus of the ICL is important for the regulation of this non-canonical Hh pathway and may have a potential application as a therapeutic intervention in NBCCS-associated KCOTs. Furthermore, the non-canonical pathway may be linked to the pathogenesis of this disease. Thus, further studies on non-canonical pathways are warranted.

\section{Acknowledgements}

This study was supported by the National Natural Science Foundation of China (grant no. 81030018) and the Doctoral Fund of the Ministry of Education of China (grant no. 20130001110043). We thank the members of our laboratory for providing the mutatation constructs (p.C727VfsX745 and p.S733IfsX736) in NBCCS-associated KCOTs. We also thank Addgene for providing the Myc-tagged cyclin B1 construct.

\section{References}

1. Gorlin RJ and Goltz RW: Multiple nevoid basal-cell epithelioma, jaw cysts and bifid rib: a syndrome. N Engl J Med 262: 908-912, 1960.

2. Gorlin RJ: Nevoid basal-cell carcinoma syndrome. Medicine (Baltimore) 66: 98-113, 1987.

3. Gorlin RJ: Nevoid basal-cell carcinoma syndrome. Dermatol Clin 13: 113-125, 1995.

4. Lo Muzio L: Nevoid basal cell carcinoma syndrome (Gorlin syndrome). Orphanet J Rare Dis 3: 32, 2008.

5. Ortega García de Amezaga A, García Arregui O,Zepeda Nuño S, Acha Sagredo A and Aguirre Urizar JM: Gorlin-Goltz syndrome: clinicopathologic aspects. Med Oral Patol Oral Cir Bucal 13: E338-E343, 2008.

6. Kiran NK, Tilak Raj TN, Mukunda KS and Rajashekar Reddy V: Nevoid basal cell carcinoma syndrome (Gorlin-Goltz syndrome). Contemp Clin Dent 3: 514-518, 2012.

7. Barreto DC, Gomez RS, Bale AE, Boson WL and De Marco L: PTCH gene mutations in odontogenic keratocysts. J Dent Res 79: 1418-1422, 2000

8. Lindström E, Shimokawa T, Toftgård R and Zaphiropoulos PG: PTCH mutations: distribution and analyses. Hum Mutat 27: 215-219, 2006.

9. Yuan JW, Li TJ, Zhong HH and Zhao HS: PTCH gene mutations in odontogenic keratocysts. Zhonghua Kou Qiang Yi Xue Za Zhi 41: 41-44, 2006 (In Chinese).

10. Li TJ, Yuan JW, Gu XM, Sun LS and Zhao HS: PTCH germline mutations in Chinese nevoid basal cell carcinoma syndrome patients. Oral Dis 14: 174-179, 2008

11. Pan S, Xu LL, Sun LS and Li TJ: Identification of known and novel PTCH mutations in both syndromic and non-syndromic keratocystic odontogenic tumors. Int J Oral Sci 1: 34-38, 2009.

12. Hahn H, Christiansen J, Wicking C, et al: A mammalian patched homolog is expressed in target tissues of sonic hedgehog and maps to a region associated with developmental abnormalities. J Biol Chem 271: 12125-12128, 1996.

13. Johnson RL, Rothman AL, Xie J, et al: Human homolog of patched, a candidate gene for the basal cell nevus syndrome. Science 272: 1668-1671, 1996.
14. Wicking C, Shanley S, Smyth L, et al: Most germ-line mutations in the nevoid basal cell carcinoma syndrome lead to a premature termination of the PATCHED protein, and no genotype-phenotype correlations are evident. Am J Hum Genet 60: 21-26, 1997.

15. Stone DM, Hynes M, Armanini M, et al: The tumour-suppressor gene patched encodes a candidate receptor for Sonic hedgehog. Nature 384: 129-134, 1996.

16. Jenkins D: Hedgehog signalling: emerging evidence for non-canonical pathways. Cell Signal 21: 1023-1034, 2009.

17. Guo YY, Zhang JY, Li XF, Luo HY, Chen F and Li TJ: PTCH1 gene mutations in keratocystic odontogenic tumors: a study of 43 Chinese patients and a systematic review. PLoS ONE 8: e77305, 2013.

18. Sun LS, Li XF and Li TJ: PTCH1 and SMO gene alterations in keratocystic odontogenic tumors. J Dent Res 87: 575-579, 2008.

19. Pan S, Dong Q, Sun LS and Li TJ: Mechanisms of inactivation of PTCH1 gene in nevoid basal cell carcinoma syndrome: modification of the two-hit hypothesis. Clin Cancer Res 16: 442-450, 2010.

20. Li TJ: Odontogenic keratocyst: A cyst, or a cystic neoplasm? J Dent Res 90: 133-142, 2011.

21. Kalderon D: Transducing the hedgehog signal. Cell 103: 371-374, 2000.

22. Evangelista M, Tian $\mathrm{H}$ and de Sauvage FJ: The hedgehog signaling pathway in cancer. Clin Cancer Res 12: 5924-5928, 2006.

23. Nurse P: Universal control mechanism regulating onset of M-phase. Nature 344: 503-508, 1990.

24. Ducommun B, Brambilla P, Félix MA, Franza BR Jr, Karsenti E and Draetta G: cdc2 phosphorylation is required for its interaction with cyclin. EMBO J 10: 3311-3319, 1991.

25. Heitlinger E, Peter M, Häner M, Lustig A, Aebi U and Nigg A: Expression of chickenlamin B2 in Escherichia coli: characterization of its structure, assembly, and molecular interactions. J Cell Biol 113: 485-495, 1991.

26. Pines J and Hunter T: The differential localization of human cyclins $\mathrm{A}$ and $\mathrm{B}$ is due to a cytoplasmic retention signal in cyclin B. EMBO J 13: 3772-3781, 1994.

27. Li J, Meyer AN and Donoghue DJ: Requirement for phosphorylation of cyclin B1 for Xenopus oocyte maturation. Mol Biol Cell 6: 1111-1124, 1995.

28. Li J, Meyer AN and Donoghue DJ: Nuclear localization of cyclin B1 mediates its biological activity and is regulated by phosphorylation. Proc Natl Acad Sci USA 94: 502-507, 1997.

29. Borgne A, Ostvold AC, Flament S and Meijer L: Intra-M phase-promoting factor phosphorylation of cyclin B at the prophase/metaphase transition. J Biol Chem 274: 11977-11986, 1999.

30. Hagting A, Jackman M, Simpson K and Pines J: Translocation of cyclin B1 to the nucleus at prophase requires a phosphorylation-dependent nuclear import signal. Curr Biol 9: 680-689, 1999.

31. Barnes EA, Kong M, Ollendorff V and Donoghue DJ: Patched1 interacts with cyclin B1 to regulate cell cycle progression. EMBO J 20: 2214-2223, 2001.

32. Barnes EA, Heidtman KJ and Donoghue DJ: Constitutive activation of the shh-ptc1 pathway by a patched 1 mutation identified in BCC. Oncogene 24: 902-915, 2005.

33. Wicking $\mathrm{C}$ and McGlinn $\mathrm{E}$ : The role of hedgehog signalling in tumorigenesis. Cancer Lett 173: 1-7, 2001.

34. Agaram NP, Collins BM, Barnes L, et al: Molecular analysis to demonstrate that odontogenic keratocysts are neoplastic. Arch Pathol Lab Med 128: 313-317, 2004.

35. Manfredi M, Vescovi P, Bonanini M and Porter S: Nevoid basal cell carcinoma syndrome: a review of the literature. Int J Oral Maxillofac Surg 33: 117-124, 2004.

36. Ingham PW and McMahon AP: Hedgehog signaling in animal development: paradigmsand principles. Genes Dev 15: 3059-3087, 2001

37. Robbins DJ, Fei DL and Riobo NA: The Hedgehog signal transduction network. Sci Signal 5: re6, 2012. 